\title{
Nettoyage ethnique, violences politiques et peuplement
}

Ethnic cleansing, political violences and settlement

Ethnische Säuberung, politische Gewalt und Besiedlung

\section{Stéphane Rosière}

\section{OpenEdition}

\section{Journals}

Édition électronique

URL : http://journals.openedition.org/rge/519

DOI : $10.4000 /$ rge. 519

ISSN : 2108-6478

\section{Éditeur}

Association des géographes de l'Est

\section{Édition imprimée}

Date de publication : 1 janvier 2005

Pagination : 5-12

ISSN : 0035-3213

\section{Référence électronique}

Stéphane Rosière, "Nettoyage ethnique, violences politiques et peuplement », Revue Géographique de l'Est [En ligne], vol. 45 / 1 | 2005, mis en ligne le 18 mai 2009, consulté le 07 septembre 2020. URL: http://journals.openedition.org/rge/519; DOI : https://doi.org/10.4000/rge.519

Ce document a été généré automatiquement le 7 septembre 2020.

Tous droits réservés 


\title{
Nettoyage ethnique, violences politiques et peuplement
}

\author{
Ethnic cleansing, political violences and settlement \\ Ethnische Säuberung, politische Gewalt und Besiedlung
}

Stéphane Rosière

\section{NOTE DE L'ÉDITEUR}

Article reçu le 17 mai 2005, accepté le 3 juin 2005

Que soient ici remerciés Messieurs Herbert Néry, Président de l'Université Nancy 2, et les professeurs Michel Roux et André-Louis Sanguin pour leur soutien concret à la réalisation de ce numéro.

1 Le but de ce numéro de la Revue Géographique de l'Est est de présenter quelques cas européens de transformation du peuplement - celui-ci étant entendu comme la répartition de la population dans l'espace - résultant de violences politiques, et particulièrement de cas de "nettoyage ethnique ». Cette problématique a une double valeur épistémologique et informative. En effet, les facteurs de distribution des hommes les plus souvent mis en avant par les géographes sont le milieu et l'économie. Les contraintes naturelles, dès $\mathrm{P}$. Vidal de la Blache au début du $\mathrm{XX}^{\mathrm{e}}$ siècle, puis les facteurs économiques sous l'impulsion de Pierre George dans les années 1950, ont été étudiés et leur influence sur le peuplement a été, à juste titre, mise en exergue. Cependant, la sous-estimation - quand ce n'est pas l'omission pure et simple - des facteurs politiques est regrettable mais perdure. Cette position paraît préjudiciable à la géographie humaine en l'écartant de débats qui traversent toutes les sciences humaines. Ainsi, ce numéro, illustré par les contributions de messieurs les Professeurs André-Louis Sanguin (Paris IV) et Michel Roux (Toulouse 2), ainsi que par de jeunes chercheurs Laurence Robin-Hunter (Paris IV) et Nicolas Lejeau (Nancy 2) concernant l'Europe du Sud-Est, soulignera l'importance des processus liés à la violence politique 
en tant que facteur agissant sur la structuration du peuplement et sur l'espace en général. Ce numéro sera aussi l'occasion de revenir sur quelques aspects méthodologiques liés à ces questions.

\section{Logiques spatiales des violences politiques}

2 La violence politique est celle qui est produite par des acteurs politiques. Dans la perspective d'une transformation violente du peuplement, on peut distinguer, en reprenant un clivage classique, l'État qui dispose en théorie du monopole de la "violence légitime» (selon l'expression célèbre de Max Weber) et la violence «illégitime ». Celle-ci est générée par des partis ou associations politiques, ou d'autres structures comme les sectes ou des Églises, puis exercée par des groupes armés qui en sont l'émanation et exercent des contraintes psychologiques et physiques pour parvenir à leurs fins. Ainsi, les groupes humains (on parlera d'ethnies, dans l'acception la plus générale et la plus neutre de ce terme) jugés indésirables peuvent-ils être victimes de déplacements ou d'expulsions forcées, voire de tentatives d'extermination de la part de nombreux acteurs.

De toute antiquité, les pouvoirs politiques ont déplacé les hommes au gré de leurs rapports plus ou moins conflictuels avec leurs sujets. Dans l'Empire ottoman, les sultans déplaçaient des ethnies (chrétiennes ou musulmanes) suivant la pratique du sürgün (littéralement le "bannissement» en turc). Il s'agissait aussi bien de peupler des secteurs stratégiques, de consolider des aires frontalières, que de punir des groupes récalcitrants. D'une manière générale, tous les grands empires ont érigé la déportation des peuples en technique de pouvoir, participant ainsi activement à la modification de la carte socioculturelle du monde.

4 Dans les États plus contemporains, la volonté d'homogénéisation des populations, suivant le principe de nationalité, est devenue le facteur déterminant. En effet, comme l'affirmait avec clarté Ernest Gellner (1989, p. 11), dès la première ligne de son ouvrage : "Le nationalisme est essentiellement un principe politique qui affirme que l'unité politique et l'unité nationale doivent être congruentes ». La recherche de l'homogénéité nationale (ou ethnique) a été pensée tout à la fois comme la condition de la légitimité et de la sécurité des États. Ainsi, beaucoup d'entre eux ont pratiqué des formes d'« aménagement du territoire " (sic) qui n'étaient pas liées à une pensée positiviste (selon le continu implicite de cette expression bien française) mais à la volonté explicite de destruction de populations allogènes, allophones ou allothrisques ${ }^{1}$. L'État, et surtout l'État-nation, a généré l'adaptation de sa population à ses normes par l'intégration forcée (la nonreconnaissance de l'altérité) ou, au contraire, par l'expulsion du corps social potentiellement, l'épuration, le «nettoyage» - qui peut viser toutes sortes de catégories de population: ethnique, culturelle, religieuse, linguistique, sociale, sexuelle, etc. (Bell-Fialkoff, 1996). On insistera évidemment ici surtout sur les clivages ethniques ou nationaux sans sous-entendre que les autres n'ont pas de pertinence.

Issues de ces logiques anciennes ou plus contemporaines, les violences politiques visant des ethnies distinctes font partie des méthodes de pouvoir utilisées dans des dizaines de pays. Ces logiques sont liées au devenir de l'État territorialisé. Elles sont liées à la création de l'État (statogenèse), à sa défense (guerre interétatique, lutte contre les séparatismes), ou à ses projets d'expansion (doctrines irrédentistes ou impérialistes). Ces logiques se sont développées au fur et à mesure que l'État a renforcé son emprise 
sur sa population, notamment par l'édification d'un encadrement administratif de plus en plus performant. L'administration, fruit et moteur de cette logique de rationalisation et d'homogénéisation, a sans cesse accru son niveau de coercition que soit par homogénéisation ethnique (par l'école et les services de l'État), ou par ingénierie ethnique (capacité à agir spatialement sur les ethnies) ou stress ethnique (pressions d'ordre législatif). Pourtant, nous ne considérerons pas que l'État soit, par essence, une structure violente, un Léviathan à l'action intrinsèquement négative nous pensons que l'État n'est que ce que l'homme veut bien en faire. Mais ce sont les représentations conflictuelles, dominantes, qui, dans une recherche d'efficacité, ont privilégié ces logiques dont la violence est l'ultime apanage. Pour autant, on doit contrebalancer ces logiques en rappelant que l'État peut (et devrait) être un garant de la sûreté de ses populations.

Dans certaines régions du monde, c'est précisément la faiblesse de l'État qui génère les violences. C'est d'abord le cas dans la périphérie du système-monde où les États "échoués ", les «Quasi-États » selon l'expression de R. Jackson (1990), ne sont plus (ou n'ont jamais été ?) en mesure de contrôler leurs territoires. L'« importation » forcée de l'État a généré un ajustement coercitif aux structures de l'État-nation pensé comme norme universelle. Cet ajustement a engendré une conflictualité structurelle dont les ethnies ne répondant pas à la norme "nationale " sont les premières et les logiques victimes. La périphérie des Empires contemporains forme aussi une autre zone de décomposition de l'État, et c'est tout particulièrement le cas de la périphérie de l'exUnion soviétique, où la faiblesse du pouvoir central génère la conflictualité. Dans ces périphéries d'empire, la violence est largement le fait de groupes ethniques, structurés en partis et/ou milices, qui se sentent (ou sont réellement) menacés et s'en prennent localement aux membres d'autres groupes, autres minorités ou groupe « majoritaire » à l'échelle de l'État. Au-delà de la rhétorique souvent réductrice de majorité/minorité (le majoritaire à l'échelle de l'État étant éventuellement minoritaire dans les régions affectées par des «nettoyages ", tel est le cas pour les Serbes au Kosovo). Dans les pays les plus multiethniques, les situations conflictuelles génèrent des affrontements croisés et complexes.

7 Cependant, on aurait tort de croire que toutes les violences politiques affectant le peuplement relèvent de l'ethnique. La violence peut être plus strictement idéologique, opposant des groupes qui appartiennent aux mêmes ethnies ou nations. La libanisation (processus d'effondrement de l'autorité de l'État et de parcellisation conflictuelle du territoire en entités séparées par des lignes de front, s'auto-administrant en toute illégalité) est une situation de violence politique liée à la faiblesse du centre. Pour ne prendre qu'un seul exemple, en Colombie, au moins trois millions de personnes auraient été déplacées depuis 1985 en raison d'un conflit dont les causes sont la compétition pour le pouvoir, l'idéologie et les narco-dollars ${ }^{2}$...

8 Ainsi, quels qu'en soient les acteurs et les formes, la tentation de la violence reste une caractéristique majeure du système-monde contemporain, et notamment de sa périphérie. Cette conflictualité structurelle se traduit par l'existence de plus de 50 millions de réfugiés et de personnes déplacées dans le monde ${ }^{3}$, la plupart étant les victimes de conflits intra-étatiques se développant en affrontements interethniques et/ ou en « nettoyages ethniques ». 


\section{Le nettoyage ethnique, une violence spécifique}

Sans revenir sur l'origine de cette expression - liée au conflit en Bosnie-Herzégovine de 1992 à 1995 (Krieg-Planque, 2003) -, ni sur l'éventail des définitions proposées (BellFialkoff, 1993 ; Roux, 2000 ; Fein, 2001 ; Mann, 2001 ; Rosière, 2004), nous partirons du postulat suivant: le "nettoyage ethnique» est une politique, et non pas une simple pratique ou une stratégie (Petrovic, 1994, p. 349), qui nécessite à la fois une pensée organisée, une idéologie et une application méthodique, par le biais de structures appropriées. Le « nettoyage ethnique " se concrétise sur le terrain grâce à une tactique articulant différents acteurs, qu'il s'agisse d'ethnocrates - suivant le néologisme de G. Aly (1999) - en tant qu'encadrement, ou d'exécutants armés. De son origine militaire, l'expression de "nettoyage» (dans le jargon militaire, "nettoyer », c'est liquider les poches de résistance dans un secteur généralement fraîchement conquis), conserve en effet une de ses caractéristiques les plus concrètes : elle est menée par une armée, sinon par la police, des groupes paramilitaires ou des milices d'autodéfense. Tous les exemples développés dans ce numéro sont dans ce cas. Le but du nettoyage ethnique est de modifier de façon durable le peuplement d'un territoire-cible. Le plus souvent, cette modification est recherchée par le départ spontané de la population (en créant du " stress ethnique »- législatif par exemple), sinon en générant un exode par le biais de pressions psychologiques de plus en plus fortes. Peu de personnes quittant spontanément leur domicile, le recours à l'expulsion manu militari est souvent nécessaire. Les violences physiques, et plus spécifiquement les massacres (Sémelin, 2004) sont un élément important dans les tactiques de «nettoyages ethniques». Plus que le massacre généralisé (ou génocide - quoique cette notion soit plus étendue), le massacre ponctuel, "exemplaire ", paraît la méthode la plus efficace en ce qu'elle engendre la fuite massive des personnes visées sans impliquer la logistique lourde que rend nécessaire une élimination systématique - dont la Shoah a été l'exemple le plus poussé. Le génocide est sans doute la violence politique la plus médiatisée, le crime de génocide a aussi valeur légale (contrairement à la notion de « nettoyage »). Le génocide (Lemkin, 1944 ; Dadrian, 1975 ; Kuper, 1981 ; Fein, 1990, 1992) est spontanément compris comme extermination. Cependant, sa définition juridique est plus large puisqu'elle implique aussi la volonté de détruire une ethnie "tout ou en partie » ${ }^{4}$. Cette notion de "partie » est interprétable en fonction de l'échelle à laquelle on analyse le phénomène. Le fait d'éliminer tous les membres d'un groupe dans un secteur donné constitue bien un acte de génocide, répréhensible à ce titre par la juridiction internationale (Dahlman, 2005). La notion de «nettoyage ethnique » a pu être critiquée (Fein, 2001) et considéré comme un euphémisme visant à couvrir des actes de génocide. Il paraît cependant possible de distinguer clairement les deux notions en posant que «si le génocide a pour finalité un peuple, le nettoyage ethnique a pour finalité un territoire" (Rosière, 2004, p. 228). Défini ainsi, le "nettoyage » s'impose comme une démarche spatiale, une géopolitique, une forme radicale de contrôle de l'espace.

\section{L'Europe : espace marqué par une forte conflictualité ethnique}

Le lien entre évolution territoriale de l'État et nettoyage ethnique atteint sans doute son paroxysme en Europe. C'est que le « vieux continent ", un des centres du système- 
monde, a inventé - à peu près en même temps que les États-Unis, mais ceux-ci dans un contexte où l'Amérindien jouait le rôle de l'indésirable - la norme de l'État-nation au peuplement homogène. Le "nettoyage » est le processus logique, rapide et violent permettant l'ajustement du peuplement à cette norme.

11 En Europe, et particulièrement dans l'Europe centrale et orientale, soit de la Grèce aux pays baltes ou à l'Ukraine, deux types de «nettoyages" peuvent se distinguer: "nettoyage » de statogenèse, et de translation territoriale (Rosière, 2004) ${ }^{5}$. Dans cet espace, la création des États s'est souvent accompagnée de « nettoyages » plus ou moins sévères (Grèce et Turquie entre 1912 et 1923 formant un cas paroxystique). Robert Hayden (1996) a fait de la partition le contexte géopolitique privilégié des «nettoyages » européens. Dans le contexte centre-européen, et plus particulièrement balkanique, les ethnies - ou nations précisément parce qu'elles prétendent former un État - sont entrées en compétition pour créer des espaces centraux de peuplement homogène. La création de ces espaces centraux a été ressentie, à tort ou à raison - il peut s'agir d'une "représentation " construite de toutes pièces autant que d'une analyse stratégique pertinente -, comme une condition nécessaire à la survie.

Dans les conflits plus récents de la fin du XXe siècle, la statogenèse s'est poursuivie par la dislocation de la Yougoslavie et de l'Union soviétique en 1991, ainsi que par la scission de la Tchécoslovaquie le 1er janvier 1993. Les "nettoyages " n'ont, bien sûr, pas été systématiques lors de ces statogenèses et ils ont concerné des États reconnus par la communauté internationale aussi bien que des entités autoproclamées : Krajina serbe de Croatie, régions serbes de Bosnie (cf. articles de Lejeau et de Robin-Hunter) ou RTCN à Chypre (cf. article de Sanguin) ; cependant, nonobstant ce distinguo juridique, ils ont alors fonctionné suivant les mêmes logiques.

D'autres cas de "nettoyage " relèvent de "translations territoriales", produites par l'accroissement du territoire de l'État. Ils concernent des territoires périphériques, en général mal intégrés à l'État, distincts du centre par leur peuplement, et de ce fait souvent en conflit avec lui. Ces politiques peuvent être menées en représailles lors de périodes d'occupation (Partisans titistes à Trieste et en Istrie en 1945, sud slovaque en 1945 également), au moment d'une annexion, ou pour mater les velléités indépendantistes de confins mal arrimés à l'entité nationale. Dans cette perspective de (re)prise en main d'une périphérie, c'est plutôt le Kosovo (cf. article de Roux) qui, dans ce numéro, s'impose comme un cas d'école, mais l'on peut aussi envisager les sécessions de la Krajina serbe et de la Republika Srpska de Bosnie-Herzégovine comme des «nettoyages de translation » liés à la volonté d'extension de la Serbie...

\section{Dans les Balkans, des effectifs considérables et un peuplement totalement remodelé}

Comme le suggère l'énumération de ces quelques exemples, les processus de "nettoyage" se sont largement épanouis dans les Balkans. Pourquoi? On doit souligner que cette région fut une des toutes premières à «importer" de manière radicale et avec le soutien des puissances occidentales, le modèle normatif de l'Étatnation (la création d'États-nations fut légèrement antérieure en Amérique latine). La création coercitive d'États-nations dans les Balkans a commencé en Grèce dans la première moitié du XIX ${ }^{e}$ siècle ${ }^{6}$. Cette statogenèse éminemment conflictuelle a eu pour corollaire massacres réciproques et expulsion des éléments allogènes dont les deux 
guerres balkaniques de 1912 et 1913 furent les épisodes les plus emblématiques (mais pas les seuls). L'espace ex-yougoslave a été concerné par des processus de ce type depuis juillet 1878 au moins (Congrès de Berlin)... et jusqu'aux guerres de 1991-95, sinon jusqu'à aujourd'hui au Kosovo... On peut donc considérer les Balkans comme le laboratoire de l'introduction de l'État-nation occidental, modèle qui s'est ensuite étendu à l'Europe centrale en 1918-19 (à cette occasion, on a d'ailleurs forgé l'expression de "balkanisation", qui n'est cependant en rien synonyme de «nettoyages»). En Europe centrale, les nettoyages ethniques ont atteint leur plus grande ampleur durant et après la Seconde Guerre mondiale. Ensuite, par le biais de la décolonisation, ils se sont imposés à l'échelle du monde, participant aux déchaînements de violences qui caractérisent le Tiers Monde contemporain.

En ce qui concerne les effectifs concernés, depuis la formation des États-nations balkaniques, le nombre de personnes déplacées, expulsées ou tuées, est considérable. Sans prétendre être exhaustif, rappelons que les guerres balkaniques ont engendré l'exil forcé de 500000 personnes (Ther, 2001, p. 49); après ces conflits, les traités bulgaro-turc de 1913 et gréco-turc de 1923 ont confirmé ou causé l'expulsion de plus de deux millions d'individus (Ladas, p. 3). Durant et après la Seconde Guerre mondiale, le « nettoyage » des minorités dans cette région s'est poursuivi. Outre la Shoah menée par les Allemands qui a participé de cette homogénéisation par la violence (la communauté juive de Roumanie est passée d'environ 900000 personnes en 1933 à 280000 en 1945 ; en Grèce d'environ 100000 en 1933, à 7000 en 1945 ; en Yougoslavie, de 70000 à 3500 après-guerre, etc.). La Yougoslavie a été le pays le plus touché par la violence contre les civils. Les massacres inter-ethniques furent d'une ampleur peu égalée durant le conflit, notamment ceux qui sont imputables au régime oustachi qui s'est lancé dans une politique génocidaire vis-à-vis des Serbes dans l'État indépendant de Croatie. Cette politique aurait causé - toutes victimes confondues, incluant aussi Juifs, Tsiganes ou autres - un peu plus d'un million de victimes... En Yougoslavie, aussi (et sans doute à cause des massacres précédents), les diverses formes de représailles d'après-guerre furent spectaculaires. Les 200000 Allemands vivant encore en Yougoslavie en 1944 furent rayés de la carte (tués ou expulsés, mais aussi la plupart des Italiens et de nombreux Hongrois). En Roumanie, en 1945, 100000 Hongrois quittèrent le pays, puis 200000 Allemands furent expulsés. Plus tard, en Bulgarie, les campagnes de «bulgarisation » du régime communiste de Jivkov ont provoqué, en 1989, le départ d'au moins 100000 Turcs (ou musulmans bulgarophones) vers la Turquie (la majorité étant rentrée depuis). La dislocation de la Yougoslavie à partir de 1991 a eu des effets considérables sur le peuplement. En Croatie, en 1991 et 1992, environ 250000 Croates ont été chassés de leurs domiciles jusqu'en 1995. Durant l'été 1995, ce sont plus de 370000 Serbes qui ont fui les régions de Croatie dont ils venaient de perdre le contrôle (cf. article de Lejeau). Bien que les déplacés Croates de 1991 aient pu rentrer chez eux, la Croatie abrite encore plus de 200000 réfugiés Croates de Bosnie-Herzégovine. Dans cette dernière république, 2,2 millions de personnes (sur une population totale de 4,2 millions en 1991) ont dû quitter leur domicile entre 1992 et novembre 1995. Le conflit a fait environ 270000 morts. Depuis les Accords de Dayton du 21 novembre 1995, la Bosnie-Herzégovine est revenue à presque 4 millions d'habitants, mais environ un million de Bosniens sont encore réfugiés de par le monde et environ 310000 sont déplacés 7 . En Serbie, on compte environ 100000 réfugiés Serbes venus de Bosnie, et 188000 de Croatie, ainsi que 225000 ayant fui le Kosovo en 1999 puis en $2004^{8}$. On peut rappeler aussi qu'au Kosovo, près d'un million d'Albanais furent temporairement 
expulsés entre 1998 et 1999, mais purent retrouver leurs foyers à partir de juin 1999. À Chypre ce sont environ 250000 personnes qui ont été déplacées après l'intervention turque de 1974 , soit près de $40 \%$ de la population de l'île...

L'ampleur de ces mouvements est donc manifeste. Une de leurs conséquences est qu'il n'existe quasiment plus une région d'Europe centre et orientale qui soit encore réellement multiethnique. À l'exception des Tsiganes, ou de reliquats de groupes ethniques autrefois plus importants, l'homogénéité nationale est aujourd'hui la règle entre l'Allemagne et la Russie (non comprise) et de la Pologne à la Grèce. Même si les grandes villes de cet espace accueillent désormais des immigrants du Tiers Monde, qui viennent à nouveau compliquer la carte ethnique, ces derniers sont rarement citoyens des pays d'accueil contrairement aux minorités nationales d'antan. L'hétérogénéité ethnique est, dans cette partie du monde, devenue l'exception, les logiques d'homogénéisation ayant eu raison de la mixité ethnique. La Croatie comptait $75 \%$ de Croates en 1991 (et $12 \%$ de Serbes), et désormais, en 2001, plus de $89 \%$ de Croates et $4,5 \%$ de Serbes. La Serbie (hors Kosovo et Voïvodine) compte désormais $85 \%$ de Serbes ; si la Bosnie apparaît dans son ensemble comme pluriethnique (elle compte près de 4 millions d'habitants à $47 \%$ Bosniaques, $39 \%$ Serbes et $13 \%$ Croates) mais à l'échelle des deux «entités » qui la compose l'hétérogénéité disparait : la Republika Srpska ( 1,4 million d'habitants) est environ à $95 \%$ serbe, et la fédération (2,3 millions) est peuplée de Bosniaques et de Croates dans les mêmes proportions - et ces deux groupes vivent en général dans des territoires distincts9. La Roumanie voisine comptait environ $71 \%$ de Roumains en 1930 , en 2002 , ce chiffre est passé à $89,5 \%{ }^{10}$. Parmi les régions de peuplement hétérogène se distinguent encore le Sandjak de Novi Pazar ou la Voïvodine (où le premier groupe, les Serbes, ne représente que $59 \%$ de la population), voire le Banat roumain. Cependant, on peut considérer que ces territoires ne sont plus que des reliquats...

On comprend donc l'ampleur terrifiante de ces « nettoyages ethniques », et leur impact psychologique, social et politique. On devine aussi les conséquences spatiales considérables du "nettoyage" dans cette région du monde, aussi bien quant à la structuration de l'espace politique que sur les pratiques spatiales de leurs habitants. Et l'on doit bien avoir à l'esprit que les Balkans ne constituent en rien un cas particulier... Cependant, on doit bien comprendre que l'homogénéisation générale des entités politiques ne relève pas non plus et strictement que du nettoyage ethnique.

\section{Le nettoyage ethnique : questions méthodologiques}

18 L'analyse raisonnée du nettoyage ethnique pose de nombreux problèmes méthodologiques : définition du corpus, connaissance de sa localisation, possibilité de comparer des recensements - si possible fondés par des maillages administratifs continus. La connaissance de ces données pose le problème de l'existence de sources fiables. Or, les situations de désagrégation de l'État sont évidemment peu propices à l'existence de sources statistiques. O'Thuatail et Dahlman (2004, p. 251) mettent ainsi en exergue le manque de recensements récents et fiables en Bosnie-Herzégovine depuis 1995, et la nécessité pour le chercheur de se baser sur les chiffres fournis par les autorités locales (communales) ou les ONG, ou les organismes internationaux. Au-delà de la question des sources, le déclin démographique d'ethnies, même lorsque a lieu un «nettoyage », ne résulte pas forcément seulement d'expulsions ou de massacres. Ainsi, 
se pose la question méthodologique de l'articulation, ou de l'imbrication, des violences politiques avec les processus démographiques et économiques.

\section{Nettoyage ethnique et dynamiques démographiques}

19 La stricte comparaison de deux recensements, lorsque l'on analyse un territoire qui a été le cadre d'un "nettoyage ", ne permet pas de connaître la part imputable aux dynamiques démographiques et celle qui l'est aux violences politiques.

Dans le cas du Kosovo, il est manifeste que les Albanais se caractérisent depuis des décennies par une natalité plus forte que celle des Serbes. Depuis les années 1950, «les Albanais ont toujours eu la natalité la plus élevée " parmi les peuples yougoslaves (Roux, 1992a, p. 151). Le taux moyen d'accroissement naturel des Albanais était de 31,1\%o (1971) et de 25,3\%o (1981), mais seulement de 6,5\% puis de 4,2 \%o pour les Serbes. Ces chiffres sont des moyennes à l'échelle de la Yougoslavie mais reflètent assez bien le différentiel du dynamisme des deux groupes au Kosovo, où même chez les Serbes, la natalité était supérieure à celle des autres Serbes (Roux, 1992a, p.152). Ainsi, les Albanais représentaient 77,3 \% de la population du Kosovo en 1981, puis 82,2\% en 1991, et sans doute $92 \%$ aujourd'hui. Le nettoyage ethnique qui a suivi la conquête du Kosovo par l'OTAN en 1999 a donc aggravé un phénomène tendant à marginaliser les Serbes, il ne l'a pas créé et le "nettoyage » est loin d'être la seule cause de l'homogénéité ethnique actuelle du peuplement de cette province.

\section{Nettoyage ethnique et dynamiques économiques}

Le «nettoyage » s'articule aussi avec des logiques économiques. Michel Roux (1992a) a ainsi souligné l'impact du sous-développement relatif du Kosovo (à l'échelle de la RFY au moins) pour expliquer la diminution de la population serbe. Les départs hors du Kosovo étaient essentiellement économiques et concernaient d'abord les Serbes. À titre d'exemple, pour la période allant du $1^{\text {er }}$ juin 1983 au 31 décembre 1987, le Kosovo a connu un solde migratoire négatif de - 22309 personnes, soit - 1,41 \% de sa population, or ce taux global était plus faible pour les Albanais $(-0,28 \%)$ que pour les Serbes ($5,69 \%$ ), seuls les Monténégrins et les Roms (respectivement $-6,57$ et $-6,06 \%$ ) connaissaient des ponctions encore supérieures (Roux, 1992a, p. 392). Cette émigration a été instrumentalisée par les Serbes, et surtout par Slobodan Milošević dans les années 1980 , dans le cadre d'une stratégie de "victimisation ». Se fondant sur l'évolution des nationalités dans la province, les Serbes accusèrent les Albanais de «purification ethnique » à leur encontre (cf. article de Roux).

Dans un autre contexte, beaucoup de Serbes vivant dans la Krajina serbe de Croatie, entre 1991 et 1995, ont dû émigrer en raison du manque de débouchés économiques dans cette république autoproclamée. La «bunkérisation » engendrée par la sécession (rupture de nombreux flux commerciaux), la diminution de la population (et donc de la demande et de l'offre) et la paupérisation entraînées par le conflit ont renforcé l'enclavement et l'anémie économique des territoires qui s'étaient autoproclamés indépendants. Cette "bunkérisation" a eu aussi des effets négatifs aussi bien en Croatie, en Bosnie-Herzégovine que dans la République Turque de Chypre Nord (la 
république de Chypre - le Sud grec - joue, comme le montre A.-L. Sanguin, de l'embargo pour faire plier, à long terme les autorités de la RTCN).

Ainsi, les dynamiques économiques engendrées par les «nettoyages» entrent fréquemment en contradiction avec la polarisation actuelle des activités dans les métropoles et sur les littoraux, et sont une cause de l'échec de ces politiques xénophobes. En effet, si ces sécessions associées à des "nettoyage» sont cohérentes d'un strict point de vue ethnique, elles sont incohérentes sinon suicidaires du point de vue économique en réduisant à la portion congrue la connectivité des territoires "nettoyés ». Ainsi, les facteurs économiques rendent généralement hasardeuse la viabilité des politiques de "nettoyage", et plus prosaïquement, en engendrant des mouvements migratoires qui ne sont pas strictement liés aux violences politiques. Ces phénomènes soulignent la nécessité d'une analyse fine des déplacements de population consécutifs à des « nettoyages ».

\section{Le nettoyage et le problème des retours}

La question des retours se pose enfin, autant d'un point de vue méthodologique que politique. Les «returnees» (suivant le terme consacré par les OIG et ONG - on utilisera ici « retournés » en français) sont les personnes expulsées, qu'elles aient été réfugiées ou déplacées, qui ont pu retrouver leur domicile. Mais le retour est une notion qui renvoie à des échelles très variables. En effet, il ne correspond pas forcément à un retour au point de départ et l'accès au foyer originel (domicile return) est parfois impossible. Le retour au sens strict devrait pourtant correspondre à la réinstallation dans le foyer d'origine, c'est en tout cas ainsi qu'il est compris, en Bosnie-Herzégovine, par l'UNHCR (O'Thuatail, Dahlman, 2004, p. 439). Si l'ancien foyer est occupé ou a été détruit, la réinstallation dans la région ou la commune d'origine forment des cas intermédiaires.

Suivant les épisodes, les retours peuvent être massifs ou marginaux, cela dépend du contexte mais aussi de l'étendue des dégâts causés par les épisodes de «nettoyage ». En juin et juillet 1999, presque tous les Albanais expulsés du Kosovo ont pu rejoindre leurs anciens domiciles; cette proportion reste beaucoup plus faible dans la Krajina serbe de Croatie ou pour les Bosniaques chassés de Republika Srpska en Bosnie-Herzégovine, elle est pratiquement nulle à Chypre.

Politiquement, la question des retours est une préoccupation centrale pour les Nations Unies. L'organisation internationale, ou ses différentes agences, combattent les politiques de "nettoyage » et tentent, autant que possible, de le rendre réversible, comme en témoigne l'annexe VII des Accords de Dayton du 21 novembre 1995. La Bosnie-Herzégovine offre en effet le cas de figure le plus emblématique, l'ONU y encourage les "retours minoritaires » au nom de son idéal affiché de multiethnicité et de tolérance. Les « retours minoritaires " sont caractérisés par le fait que le retourné, d'une ethnie " $\mathrm{x}$ », cohabite avec une autre ethnie "y» localement majoritaire. Le retour minoritaire n'implique pas systématiquement un vrai domicile return... Laurence Robin-Hunter souligne les aspects contradictoires des accords de Dayton et les résultats mi-figue, mi-raisin de cette politique. Cependant, en Bosnie-Herzégovine, les retours minoritaires concernaient environ 450000 personnes en décembre 2004, ce qui n'est pas négligeable. 
27 Au-delà du cas de la Bosnie-Herzégovine, le nombre réel de retours est souvent inférieur aux chiffres fournis par le HCR. Une part significative des retournés minoritaires ne reviennent que pour vendre leurs biens, ou restent quelque temps mais repartent découragés (chômage, pressions psychologiques, problème pour l'éducation des enfants, etc.), ou parce qu'ils craignent pour leur vie. Durant l'année 2004, l'UNHCR décomptait ainsi 135 incidents, allant jusqu'au meurtre, sur des « retournés » en Bosnie (56 dans la république serbe, 73 dans la fédération croato-bosniaque et 6 à Brčko) ${ }^{11}$. Finalement, les retours durables sont assez souvent le fait de personnes âgées. L'âge moyen élevé des retournés vient aussi du fait que les jeunes adultes ont plus de facilité à s'installer définitivement dans un pays d'accueil, mais aussi parce que le «nettoyage » a donné le coup de grâce à de nombreuses activités économiques et que le retour n'offre aucune perspective économique.

Les retours durables au pays d'origine se font souvent dans un lieu différent de celui dont on a été expulsé. Les retours majoritaires se font rarement vers les campagnes. Ils contribuent au contraire à l'exode rural dans des régions qui restent souvent ravagées, avec des terrains minés, une voirie ou des services hors d'état, et des perspectives économiques inexistantes. La ville est alors le lieu du seul salut, celui où se concentrent à la fois les logements, le travail sinon les aides internationales. Ainsi, malgré l'existence de retours ponctuels, le nettoyage ethnique contribue-t-il au dépeuplement des campagnes, au vieillissement des populations rurales et à la désertification économique de ces espaces.

Finalement, le nettoyage ethnique s'impose comme un processus majeur, un élément fondamental de la structuration de l'espace aussi bien dans les Balkans que dans l'Europe orientale en général (triangle Pologne, Grèce, Russie). En mettant en exergue le nettoyage ethnique et les violences politiques comme facteurs structurant de l'espace, la Revue Géographique de l'Est espère contribuer à la rénovation des problématiques de géographie humaine. Une approche plus post-moderne, qui tienne compte à la fois des violences politiques et des victimes, permet en effet d'appréhender la structuration de l'espace avec une plus grande acuité.

\section{BIBLIOGRAPHIE}

Cette bibliographie dépasse largement les seules références du texte ci-dessus. Elle a pour but d'offrir au chercheur une base de données sur le double thème des violences politiques et du nettoyage ethnique. Elle intègre donc des références théoriques générales, mais aussi des études de cas concernant l'Europe orientale d'une manière générale.

Aly G. (1999). - Final Solution, Nazi Population Policy and the Murder of the European Jews, Londres, Arnold, 295 p.

BADIE B. (1992). - L'État importé. L'occidentalisation du monde, Paris, Fayard, 334 p.

BALIBAR É., WALLERSTEIN I. (1997). - Race, nation, classe. Les identités ambiguës, Paris, La Découverte, collection Poche, 308 p. 
BAUMAN Z. (1989). - Modernity and the Holocaust, Ithaca, Cornell University Press [en français, (2002), Modernité et holocauste, Paris, La Fabrique, 285 p. dans une traduction « légèrement abrégée » selon les termes de l'éditeur].

Bell-FialKoff A. (1993). - « A Brief History of Ethnic Cleansing », New York, Foreign Affairs, vol. 72, $n^{\circ} 3$, pp. 110-121.

Bell-FialKoff A. (1996). - Ethnic Cleansing, New York, Londres, St Martin's Press, Mac Millan, 346 p.

BREUILLY J. (2001). - « The State and Nationalism ». In : Guibernau M., Hutchinson J., Understanding Nationalism, Cambridge, Polity Press, pp. 32-52.

CAMBRÉZy L. (2001). - Réfugiés et exilés. Crise des sociétés, crise des territoires, Paris, éditions des Archives Contemporaines, $216 \mathrm{p}$.

CARMichael C. (2002). - Ethnic Cleansing in the Balkans. Nationalism and the Destruction of Tradition, Londres, New York, Routledge, $173 \mathrm{p}$.

CHARNY I., dir. (2001). - Le livre noir de l'humanité. Encyclopédie mondiale des génocides, Toulouse, Privat, $720 \mathrm{p}$.

CIGAR N. (1995). - Genocide in Bosnia : the Policy of " ethnic cleansing »,College Station, Texas A \& M University Press, $242 \mathrm{p}$.

DADRIAN V. N. (1975). - « A Typology of Genocide », Toronto, International Review of Modern Sociology, $\mathrm{n}^{\circ}$ 5, pp. 201-212.

DADRIAN V. N. (1996). - Histoire du génocide arménien. Conflits nationaux des Balkans au Caucase, Paris, Stock, $693 \mathrm{p}$.

Dahlman C. (2005). - « Geographies of Genocide and Ethnic Cleansing : The Lessons of BosniaHerzegovina ». In : Flint C., ed., The Geography of War and Peace, Oxford, New York, Oxford University Press, pp. 174-198.

DRoBizHeVA L., et al. (1996). - EthnicConflict in the Post-Soviet World, Cases Studies and Analysis, New York, Londres, Sharpe, Armonk, XV-365 p.

ELSIE R., ed. (2002). - Gathering Clouds. The Roots of Ethnic Cleansing in Kosovo and Macedonia, Prishtinë, $172 \mathrm{p}$.

FEIN H. (1984). - «Scenarios of Genocide : Models of Genocide and Critical Responses », in :Toward the Understanding and Prevention of Genocide, Proceedings of the International Conference on the Holocaust and Genocide, Israel W. Charny, ed. Boulder (CO), Westview Press, pp. 3-31.

FEIN H. (1990). - « Genocide : a Sociological Perspective », Loughborough, Current Sociology, vol. $38, \mathrm{n}^{\circ} 1$, pp. 104-116.

FeIN H., ed. (1992). - Genocidal Watch, New Haven, Yale University Press, X-204 p.

FEIN H. (2001). - Ethnic Cleansing and Genocide : Definitional Evasion, Fog, Morass or Opportunity?, Minneapolis (MN), Association of Genocide Scholars Conference.

GELLNER E. (1989). - Nations et nationalismes, Paris, Payot, collection « Bibliothèque historique ", 208 p. [1re éd., 1983].

GRMEK M., GJIDARA M., SIMAC N. (1993). - Le nettoyage ethnique. Documents historiques sur une idéologie serbe, Paris, Fayard, 340 p. 
GUIBERNEAU M., HUNTCHINSON J., eds. (2001). - Understanding Nationalism, Cambridge, Polity Press, $273 \mathrm{p}$.

HAYDEN R. M. (1996). - « Schindler's Fate : Genocide, Ethnic Cleansing and Population Transfers », Champaign (IL), Slavic Review, vol. 55, $\mathrm{n}^{\circ}$ 4, pp. 727-748.

HARFF B., GURR T. R. (1988). - « Toward Empirical Theory of Genocides and Politicides :

Identification and Measurement of cases since 1945 ", Denton (TX), International Studies Quarterly, $\mathrm{n}^{\circ} 32, \mathrm{pp} .359-371$

HARFF B. (2003). - « No Lessons Learned from the Holocaust? Assessing Risks of Genocide and Political Mass Murder since 1955 ", Washington, American Political ScienceReview, vol. 97, n 1, pp. 57-73.

HASSNER P. (2000). - La violence et la paix: de la bombe atomique au nettoyage ethnique, Paris, Le Seuil, $370 \mathrm{p}$.

JACKSON R. H. (1990). - Quasi-states, Sovereignety, International Relations and the Third-World, Cambridge, New York, Cambridge University Press, X-225 p.

KIERNAN B. (1999). - « Sur la notion de génocide », Paris, Le Débat, n 104, pp. 179-192.

KIERNAN B., GELlately R., eds. (2003). - Comparative Genocides, Cambridge, New York, Cambridge University Press, 390 p.

KRIEG-PlANQUe A. (2003). - Purification ethnique. Une formule et son histoire, Paris, CNRS éditions, $528 \mathrm{p}$.

KUPER L. (1981). - Genocide and its political use in the XX ${ }^{\text {th }}$ Century, Harmondsworth, New York, Penguin Books, 255 p.

LADAS S. (1932). - The Exchange of Minorities : Bulgaria, Greece and Turkey, New York, Mac Millan Company, $849 \mathrm{p}$.

LAITIN D. (1995). - « Ethnic Cleansing, Liberal style », Cambridge (MA), McArthur Foundation Program in Transnational Security, Working Paper Series, $\mathrm{n}^{\circ} 4$.

LE COUR GRANDMAISON O. (2005). - Coloniser, exterminer. Sur la guerre et l'État colonial, Paris, Fayard, $360 \mathrm{p}$.

LEMBERG H. (1992). — « Ethnische Säuberung : Ein Mittel und Lösung von Nationalitäten problemen? », Bonn, Aus Politik und Zeitgeschichte, vol. 46, pp. 27-38.

LEMKIN R. (1944). - Axis Rules in Occupied Europe, Washington DC, Carnegie Endowment for World Peace.

LEPAGE D., KULLASHI M., dir. (2002). - Ex-Yougoslavie : une Europe du Sud-Est en construction, Paris, L'Harmattan, $168 \mathrm{p}$.

LEVENE M., ROBERT P., eds. (1999). - The Massacre in History, New York, Oxford, Berghahn Books.

Livre noir de l'ex-Yougoslavie, Purification ethnique et crimes de guerre (1993). - Paris, Arléa, Documents rassemblés par Le Nouvel Observateur et Reporters Sans Frontières, 487 p.

Mann M. (1999). - « The Dark Side of Democracy : The Modern Tradition of Ethnic and Political Cleansing ", Londres, The New Left Review, n² 235, pp. 18-45.

MANN M. (2001). - « Explaining Murdrous Ethnic Cleansing : the Macro-level ». In : Guiberneau M., Huntchinson J. - eds., Understanding Nationalism, Cambridge, Polity Press, pp. 207-241. 
MANN M. (2005). - The Dark Side of Democracy. Explaining Ethnic Cleansing, Cambridge, New York, Cambridge University Press, $450 \mathrm{p}$.

MARConi S. (2000). - «Etnia, razza e "pulizia" ", Rome, Critica Sociologica, n 134, pp. 48-64.

MARRUS M. R. (1985). - The Unwanted. European Refugees in the Twentieth Century, New York, Oxford, Oxford University Press, XII-414 p.

MARTIN T. (1998). - « The Origins of Soviet Ethnic Cleansing », Chicago, The Journal of Modern History, $\mathrm{n}^{\circ} 70$, pp. 817-824.

MARTIN T. (2001). - The Affirmative Action Empire. Nations and Nationalism in the Soviet Union, 1923-1939, Ithaca (NY), Cornell University Press, 496 p.

Meindersma C. (1997). - « Population Exchanges, Law and State Practice », Oxford, International Journal of Refugee Law, Part 1, vol. 9, n 3, pp. 335-364 ; Part 2, vol. 9, n 4, pp. 613-653.

Michels P. (1997). - « Le discours sur le nettoyage ethnique : comment diaboliser une nation », Paris, Revue d'Études Comparatives Est-Ouest, vol. 28, n 1, pp. 147-162.

NAIMARK N. (2001). - Fires of Hatred, Ethnic Cleansing in Twentieth-Century Europe, Cambridge (MA), Londres, Harvard University Press, 248 p.

O’thuATAil G., DAHLMAN C. (2004). - « The Effort to Reverse Ethnic Cleansing in Bosnia-

Herzegovina : The Limits of Returns ", Columbia (MD), Eurasian Geography and Economics, vol. 45, $\mathrm{n}^{\circ}$ 6, pp. 439-464.

Petersen W. (1958). - « A General Typology of Migration », Philadelphie (PA), American Sociological Review, vol. 23, $\mathrm{n}^{\circ} 3$.

Petrovic D. (1994). - «Ethnic Cleansing : An Attempt at Methodology », Florence, European Journal of International Law, vol. 5, n 3, pp. 342-359.

PoHL J. O. (1999). - Ethnic Cleansing in the USSR, 1937-1949, Wesport (CT), Londres, Greenwood Press, $179 \mathrm{p}$.

RoSIÈRE S. (2004). - « Le nettoyage ethnique - approche géographique », Bâle, Geographica Helvetica, $\mathrm{n}^{\circ} 3$, pp. 227-237.

Roux M. (1992a). - Les Albanais en Yougoslavie. Minorité nationale, territoire et développement, Paris, Éditions de la Maison des Sciences de l'Homme, $546 \mathrm{p}$.

Roux M. (1992b). - « A propos de la purification ethnique en Bosnie-Herzégovine », Paris, Hérodote, $n^{\circ} 67$, pp. 49-60.

Roux M. (2000). - « Nettoyage ethnique ». In : Cordellier S. - dir., Dictionnaire historique et géopolitique du 20e siècle, Paris, La Découverte, pp. 484-486.

SANGUIN A.-L. (1998). - « Retour à Srbrenica, notes de terrain à propos d'un nettoyage ethnique devenu génocide culturel », Paris, Géographie et Cultures, n² 27, pp. 63-78.

SANGUIN A.-L. (2001). - « La Bosnie, État tricéphale des Balkans. Les processus d'une partition ethno-politique », Paris, Géographie et Cultures, $\mathrm{n}^{\circ} 38$, Conflits et minorités dans l'Europe postcommuniste, pp. 65-84.

SCHEChTMAN J. B. (1971). - European Populations Transferts (1939-45), New York (NY), Russel \& Russel [1 $1^{\text {ère }}$ éd., Oxford, 1946].

SÉMELIN J. (2002). - « Du massacre au processus génocidaire », Paris, Revue Internationale des Sciences Sociales, $\mathrm{n}^{\circ}$ 174, pp. 483-492 [sur le site du CERI : www.ceri-sciences-po.org]. 
SÉMELIN J. (2003). - « Analysis of a Mass Crime. Ethnic Cleansing in the Former Yougoslavia (1991-1999) ». In : Kiernan B., Gellately R. - eds. Comparative Genocides, Cambridge, New York, Cambridge University Press, pp. 353-373.

SÉMELIN J. (2004). - « Éléments pour une grammaire du massacre », Paris, Le Débat, n 124, pp. 154-170.

STAUB E. (1989). - The Roots of Evil : the Origin of Genocide and other Group Violence, Cambridge, New York, Cambridge University Press.

TERNON Y. (1995). - L'État criminel. Les génocides au XXe siècle, Paris, Le Seuil, 449 p.

THER PH., SILJAK A., eds. (2001). - Redrawing Nations, Ethnic cleansing in East-Central Europe, 1944-1948, Lanham (MD), Rowman \& Littlefield Publishers Inc., 340 p.

VANDENBERGHE F. (1996). - « Légalité, légitimité et politique du nettoyage ethnique », Caen, Mana, revue de sociologie et d'anthropologie, $\mathrm{n}^{\circ} 2$, pp. 193-216.

VARDY S. B., TOOLEY T. H., eds. (2003). - Ethnic Cleansing in 20th-Century Europe, Boulder (CO), Social Science Monographs, $860 \mathrm{p}$.

YÉRASIMOS S. (1993). - Questions d'Orient, Frontières et minorités des Balkans au Caucase, Paris, La Découverte, collection « Livres Hérodote », 246 p.

WEINER A., ed. (2003). - Landscaping the Human Garden, Twentieth-Century Population Management in a Comparative Framework, Palo Alto (CA), Stanford University Press, 344 p.

\section{NOTES}

1. Allogène: d'une autre origine; allophone: d'une autre langue; allothrisque: d'une autre religion.

2. Chiffre fourni par le Comité International de la Croix-Rouge, du 22 avril 2005. Lien : http:// www.icrc.org/Web/fre/sitefre0.nsf/iwpList516/BF2BCC541D8A22B5C1256FD600512EA6

3. Les réfugiés sont des personnes qui fuient leur pays d'origine et s'installent dans un autre pays, limitrophe ou plus lointain. Les réfugiés sont souvent regroupés dans des camps établis dans les régions frontalières de leur pays d'origine. Plus tardivement prises en compte par la communauté internationale, les personnes déplacées ont, elles aussi, quitté leur domicile, mais elles restent à l'intérieur des frontières du pays dont elles sont ressortissantes. Réfugiés et déplacés sont souvent regroupés dans des camps.

4. Selon la Convention pour la prévention et la répression du crime de génocide adoptée le 9 décembre 1948, le génocide est défini comme acte "commis dans l'intention de détruire, tout ou en partie, un groupe national, ethnique, racial ou religieux».

5. On peut désigner par «translation territoriale » tout déplacement de frontière n'aboutissant ni à la création, ni à la disparition d'un État. Ce processus simple se caractérise donc par l'agrandissement du territoire d'un État et la diminution du territoire d'un autre.

6. L'indépendance de la Grèce est proclamée à Epidaure en 1822, mais les Ottomans ne la reconnaissent qu'en 1832 par le traité de Constantinople.

7. UNHCR, « Update on Conditions for Return to Bosnia and Herzegovina », janvier 2005.

8. Chiffres de la CIA, du 10 février 2005, disponibles via le lien: http://www.cia.gov/cia/ publications/factbook/geos/yi.html

9. www.ined.fr

10. Hors des Balkans, l'archétype de l'homogénéisation serait sans doute la Pologne qui comptait environ $70 \%$ de Polonais selon son recensement de 1921, et 96,7 \% - sur un territoire différent - 
en 2002. À moins que l'on ne désigne la Prusse-orientale qui a perdu deux fois toute sa population originelle. Une première fois, lors de la colonisation allemande médiévale, par extermination et assimilation des Vieux-Prussiens, puis entre 1944 et 1948 par expulsion des Allemands et colonisation soviétique.

11. UNHCR, Update on Conditions for Return to Bosnia and Herzegovina, January 2005, p. 5.

\section{RÉSUMÉS}

Cet article introductif souligne le rôle structurant de la violence politique sur le peuplement. La violence politique est souvent le fait des États, mais elle peut aussi résulter de leur faiblesse. Parmi les différentes formes de violences politiques exercées à l'encontre des populations, le nettoyage ethnique est particulièrement lié à la création d'États ou à leur évolution spatiale. La recherche de l'homogénéité ethnique engendre logiquement des processus de modification du peuplement par la violence. Ces processus peuvent être compris comme des formes d'ajustements structurels à la norme de l'État-nation homogène. L'Europe en général et les Balkans en particulier ont été particulièrement concernés par ces processus de la fin du XIX ${ }^{\mathrm{e}}$ siècle jusqu'à nos jours. L'approche méthodologique du nettoyage ethnique met en exergue plusieurs problèmes: définition du corpus, connaissance de sa localisation, possibilité de comparer des recensements. Le traitement de ces données pose le problème de l'existence de sources fiables. Les retours éventuels de personnes chassées, après les épisodes de "nettoyage ", génèrent de nouvelles difficultés dans l'appréciation de ces processus puisqu'il est en effet souvent difficile de déterminer si les retours sont définitifs.

This paper underlines how political violence explain settlement and population location. Political violence is produced by states or by the weakness of the states. Among the various forms of violence, ethnic cleansing is especially linked with state creation or its territorial transformation. Indeed, the research of ethnical homogeneity logically generates violent processes of settlement transformation. These processes may be considered as structural adjustments to the criterion of the homogeneous Nation-State. Europe in general, and Balkans especially are very much concerned by these violent processes since the end of the nineteenth century up until now. The methodological approach of ethnic cleansing emphasizes different problems: definition of corpus, knowledge of the settlement, possibility to compare it before and after the events. These elements imply reliable information that unstable situations do not provide. Furthermore potential returns (of refugees or displaced persons) generate specific difficulties, which vary according to the nature of the return, i.e. permanent or not.

Dieser einleitende Artikel unterstreicht die Rolle der politischen Gewalt in der Besiedlung. Die politische Gewalt ist meist die Tatsache der Staaten, aber sie kann auch durch ihre Schwäche verursacht werden. Unter den verschiedenen Formen der politischen Gewalt gegen die Bevölkerungen ist die ethnische Säuberung besonders in Verbindung mit der Entstehung oder der Raumentwicklung von Staaten. Die Sehnsucht nach einer ethnischen Homogenisierung verursacht Veränderungsprozesse der Bevölkerung durch die Gewalt. Diese Prozesse entsprechen einer Anpassung zu der einheitlichen Staatsnation. Seit dem Ende des 19. Jhs bis heute wurde Europa und insbesondere Balkan von diesen Prozessen betroffen. Die Methodologie zur Forschung über die ethnische Säuberung stellt mehrere Probleme voran: Charakterisierung 
des Korpus, Kenntnis seiner Lage, Möglichkeit eines Vergleiches der Volkszählungen. Die Bearbeitung dieser Angaben stellt das Problem von dem Vorhandensein sicherer Quellen. Der Rückkehr der Vertriebenen nach der Zeit der Säuberung verursacht neue Schwierigkeiten um die Einschätzung dieser Prozesse, da es meist schwierig ist, einzuschätzen, ob der Rückkehr endgültig ist.

\section{INDEX}

Keywords : Balkans, demography, ethnic cleansing, Europe, Nation-State, settlement, State, violence

Schlüsselwörter : Balkan, Besiedlung, Demographie, ethnische Säuberung, Europa, Gewalt, Staaten, Staatsnationen

Mots-clés : Balkans, démographie, État, État-nation, Europe, nettoyage ethnique, peuplement, violence

\section{AUTEUR}

\section{STÉPHANE ROSIÈRE}

Maître de conférences habilité, Université Nancy 2, Département de Géographie, CERPA, BP 33-97, F-54015 Nancy Cedex, France, stephane.rosiere@univ-nancy2.fr 